\title{
Evaluación de la fase coloidal de un suelo Chromic Dystedents a la aplicación de cachaza madura panelera
}

\section{Evaluation of colloidal face in a Chromic Dystrudents soil to application of mature sugar cane sludge}

\author{
Fabio E. Forero ${ }^{1 *}$, Karen V. Suárez², Nicolás Forero ${ }^{3}$
}

Recibido para publicación: Agosto 8 de 2016 - Aceptado para publicación: Abril de 2017

\begin{abstract}
RESUMEN
Las aplicaciones de enmiendas orgánicas son alternativas para el mejoramiento físico, químico y biológico de los suelos, un buen ejemplo es la cachaza madura o fresca, subproducto del proceso artesanal o semi industrial de producción de panela en la región de la Hoya del Rio Suárez, Chitaraque, Boyacá. En esta investigación se determinó el comportamiento de las bases intercambiables calcio $\left(\mathrm{Ca}^{+2}\right)$, Magnesio $\left(\mathrm{Mg}^{+2}\right)$ y potasio $\left(\mathrm{K}^{+}\right)$en la fracción coloidal del suelo. Se determinaron las concentraciones de las bases antes de la siembra y en la cosecha del cultivo de frijol variedad ICA Cerinza; se utilizó un diseño completamente al azar con seis tratamientos T0: Testigo solo con enmienda calcárea $\left(6,6 \mathrm{t} \mathrm{ha}^{-1}\right.$ Dolomita). T1: comercial $\left(0,44 \mathrm{tha}^{-1}\right.$ de triple quince + 6,6 tha-1 Dolomita); T2: Cachaza madura (5,7 t ha1); T3: Abimgra (3,3 t ha-1); T4: 75\% Cachaza madura + Abimgra (4,27 t ha ${ }^{-1}$ más 0,25 t ha $\left.{ }^{-1}\right)$. T5: 50\% Cachaza $+50 \%$ de Abimgra (2,85 t ha ${ }^{-1}$ más 1,65 $\left.\mathrm{t} \mathrm{ha}^{-1}\right)$; T6: $25 \%$ Cachaza madura $+75 \%$ Abimgra, Los resultados mostraron que T5 mostro diferencias significativas y que con la aplicación de 2,85 tha-1 de Cachaza y 1,65 t ha ${ }^{-1}$ de Abimgra se obtienen los mejores resultados en el incremento de las bases $\mathrm{Ca}^{+2}$ y $\mathrm{Mg}^{+2}$ Ilegando a valores de $7 \mathrm{cmol}^{(+)} \mathrm{kg}^{-1}$ de suelo y $1,8 \mathrm{cmol}(+)$ $\mathrm{kg}^{-1}$ de suelo respectivamente; concluyendo que la aplicación de cachaza madura en mezcla posee gran potencial de aporte de Calcio y magnesio.
\end{abstract}

Palabras clave: Solución del suelo, capacidad de Intercambio catiónico, saturación, mineralización, abono orgánico.

\begin{abstract}
The applications of organic modifications are alternatives used for the physical, chemical and biological improvement of soil. A good example is mature or fresh sugar cane sludge, a by-product of the traditional or semi-industrial process of the sugarcaneproducing region called "Hoya del Río Suárez (Chitaraque, Boyacá)". In this research, the behavior of the interchangeable bases Calcium $\left(\mathrm{Ca}^{+2}\right)$, Magnesium $\left(\mathrm{Mg}^{+2}\right)$ and Potassium $\left(\mathrm{K}^{+}\right)$in the colloidal fraction of the soil was determined from a DCA type design with six treatments (TO: Control only with calcareous modification (6.6 t ha-1 Dolomite). T1: Commercially (0.44 $\mathrm{t} \mathrm{ha}^{-1}$ of 15-15-15) + Dolomite. T2: mature sugar cane sludge (5.7 $\left.\mathrm{t} \mathrm{ha}^{-1}\right)$. T3: Abimgra $\left(3,3 \mathrm{t} \mathrm{ha}^{-1}\right)$. T4: $75 \%$ mature sugarcane sludge + Abimgra $\left(2.85 \mathrm{t} \mathrm{ha}^{-1}\right.$ plus $\left.1.65 \mathrm{t} \mathrm{ha}^{-1}\right)$. T5: $50 \%$ sugarcane sludge $+50 \%$ Abimgra (2.85 t ha-1 plus $\left.1.65 \mathrm{t} \mathrm{ha}^{-1}\right)$. T6: 25\% mature sugarcane sludge $+75 \%$ Abimgra (1.42t ha ${ }^{-1}$ plus 2.47 $\mathrm{t} \mathrm{ha}^{-1}$ ) and four replications where the concentrations of the bases were determined before sowing and in the crop of the Cerinza ICA variety bean. Concluding that the application of $2.85 \mathrm{tha}^{-1}$ of sugar cane sludge and 1.65 td ha-1 of Abimbra, obtained the best results in the increase of $\mathrm{Ca}^{+2}$ and $\mathrm{Mg}^{+2}$ bases, reaching values of $7 \mathrm{cmol}^{(+)} \mathrm{kg}^{-1}$ of soil and $1.8 \mathrm{cmol}(+) \mathrm{kg}-1$ of soil respectively. The above stated explains the great potential of mature sugar cane sludge with the contribution of calcium and magnesium.
\end{abstract}

Keywords: Soil solution, Cation exchange capacity, Saturation, Mineralization, organic fertilizer.

\footnotetext{
${ }^{1 *}$ M.Sc. Docente FACIAT, Universidad Pedagógica y Tecnológica de Colombia. Tunja - Colombia. guatoquero@gmail.com

${ }^{2}$ M.Sc. Universidad Pedagógica y Tecnológica de Colombia. Tunja - Colombia. vickoaf@gmail.com

${ }^{3}$ Estudiante Biología, Universidad Pedagógica y Tecnológica de Colombia. Tunja - Colombia. nicolas.forero@uptc.edu.co
} 


\section{INTRODUCCIÓN}

Dentro de la actividad panelera artesanal y semi industrial en la Hoya del Río Suárez se desprende un subproducto de alto impacto económico y subutilizado conocido como Cachaza o Torta de filtro, la cual es el resultado de los sólidos desechados en la etapa de clarificado dentro del proceso de producción panelera (Gómez y Jaramillo 2006). A pesar de considerarse un subproducto que genera un impacto negativo en el ambiente como exceso de producción de metano, olores desagradables, proliferación de moscas, roedores, gallinazos y culebrillas; se ha encontrado en diversos estudios su aplicabilidad como fuente de elementos nutricionales para el desarrollo de la actividad agrícola, especialmente por el aporte de Ca y P (Arreola-Enriquez et al. 2004); sin embargo, muchos autores referencian que el contenido nutricional del producto depende directamente de la zona de producción y la forma de procesamiento (Cifuentes et al. 2011).

Los desechos generados de la industria azucarera tienen alto contenido de materia orgánica, que después de ser estabilizados biológicamente (descomposición) presentan características similares a las de la materia orgánica del suelo, por tanto, tiene alto potencial como acondicionador que permite aportar cantidades altas de humus necesarias para mantener la fertilidad de suelos en zonas tropicales y subtropicales (Mohee y Panray 1999).

La cachaza es considerada como el subproducto más importante de los ingenios azucareros y en la agroindustria artesanal de la caña panelera en la producción de panela, con algún valor como fertilizante, produciéndose hasta $3 \mathrm{t}$ húmedas, por cada 100 t de caña molida; es un material marrón oscuro, constituido por una mezcla de fibra de caña, sacarosa, coloides, coagulados, incluyendo la cera, fosfato de calcio y partículas de suelo (Zurro 2005).
La cachaza se recoge a la salida de los filtros al vacío, presentando aproximadamente un $25 \%$ de materia seca. Este material contiene muchos de los coloides de la materia orgánica originalmente dispersa en el jugo, conjuntamente con aniones orgánicos e inorgánicos que precipitan durante la clarificación (Gómez 2006); sin embargo, en la Hoya del Río Suárez (Chitaraque, Boyacá), por ser un proceso artesanal, la clarificación se realiza mediante el uso de prelimpiadores que son pequeños tanques en ladrillo o concreto ubicados después del molino.

Las propiedades de la Cachaza o Torta de filtro no solamente van en el sentido químico, puesto que se ha reconocido internacionalmente la capacidad que tiene el producto en el mejoramiento de las propiedades físicas, químicas y biológicas del suelo, a pesar del contenido de humedad del material que oscila entre un $46 \%$ a $70 \%$ (Forero et al. 2008). Tradicionalmente, la cachaza ha sido considerada un subproducto panelero que posee problemas de manejo, almacenamiento, alto contenido de $\mathrm{CH}_{4}$ y $\mathrm{CO}_{2}$, malos olores, efecto fitotóxico en múltiples cultivos, lo que conlleva a la generación de problemas de toxicidad en las plantas; por ello, la necesidad constante de reconocer y plantear nuevas estrategias de manejo, para el aprovechamiento del subproducto que permita mantener el equilibro ambiental y económico en el proceso panelero regional (Bernal et al. 2013).

Según Forero et al. (2008), la cachaza que proviene del área panelera de la Hoya del Rio Suárez, ubicada en los departamentos de Boyacá y Santander, tiene el potencial de aportar en promedio $\mathrm{Ca}(6,52 \%), \mathrm{K}(0,73 \%), \mathrm{Mg}$ $(0,66 \%)$ y $N(1,15 \%)$ y al igual un alto potencial de aporte de materia orgánica $(21,1 \%$ ) a la cual se le atribuye principalmente la reducción de la densidad aparente del suelo y el aumento en la estabilidad estructural, generando suelos estables capaces de sostener cualquier tipo de 
actividad en el mismo, lo cual le permite en el corto tiempo ser menos susceptible a los agentes erosivos (Montaño y Forero 2013).

Treto (2005) en La Habana manifiesta la importancia de la utilización de la cachaza o torta de filtro como fuente orgánica de la industria azucarera desde 1917 a la fecha, reportando resultados en caña de azúcar y otros cultivos, destacándola como un producto fertilizante de interés, por su alto contenido de fósforo, calcio, nitrógeno y materia orgánica; igualmente, reporta que en caña de azúcar la cachaza puede suplir toda la fertilización química durante tres años en suelos arenosos y en suelos arenosos sílicos como promedio hasta de cinco años con dosis de $120 \mathrm{t} \mathrm{ha}^{-1}$ a $160 \mathrm{t}$ ha-1 y 180 t ha $^{-1}$ a 240 t ha $^{-1}$ respectivamente.

Como la aplicación de cachaza en suelos agrícolas, es una alternativa para el manejo del producto y una fuente de abono orgánico para la fertilización de caña panelera, que por producirse en las unidades productoras artesanales (trapiches) no generan costos adicionales en lo referente a transporte, permitiendo un alto grado de rentabilidad en el uso del subproducto.

El objetivo principal de esta investigación consistió en la aplicación de cachaza madura en mezcla con Abimgra (abono orgánico - mineral), con el fin de evaluar el comportamiento de las bases $\mathrm{Ca}^{+2}, \mathrm{Mg}^{+2}$ y $\mathrm{K}^{+}$ en un Vertisol del Municipio de Chitaraque, departamento de Boyacá.

\section{MATERIALES Y MÉTODOS}

El estudio fue desarrollado durante los meses de marzo a septiembre de 2014, en el Municipio de Chitaraque, Vereda Tume Chico, Finca el Anacal (Boyacá), ubicada a $6^{\circ} 00^{\prime} 29^{\prime \prime} \mathrm{N}$ y $73^{\circ} 28^{\prime} 32^{\prime \prime} \mathrm{W}$, con una altura de 1800 msnm. Las condiciones climáticas de la zona durante el periodo del experimento en campo (cuatro meses) fueron las siguientes: precipitación $660 \mathrm{~mm}$, temperatura promedio de $18,6{ }^{\circ} \mathrm{C}$, temperatura máxima promedio de $25,9{ }^{\circ} \mathrm{C}$ y mínima promedio de $13{ }^{\circ} \mathrm{C}$ y humedad relativa promedio del $80 \%$.

Se utilizó un diseño completamente al azar (debido a la homogeneidad en pendiente del lote utilizado en el ensayo), con siete tratamientos y cuatro repeticiones, la unidad experimental de $4 \mathrm{~m}$ de largo por $6 \mathrm{~m}$ de ancho para un área $24 \mathrm{~m}^{2}$ con área de $960 \mathrm{~m}^{2}$, dentro de cada parcela se aplicaron las dosis referenciadas en la tabla 1 y utilizando como cultivo indicador el frijol así: distancia entre surcos de $70 \mathrm{~cm}$ (5 surcos), dejando $20 \mathrm{~cm}$ de borde de la unidad experimental y entre plantas $20 \mathrm{~cm}$ (28 sitios), dejando $20 \mathrm{~cm}$ de desborde, 140 sitios de siembra en la parcela, colocando tres granos por sitio en total 420 semillas, para una población de 7840 plantas.

Tabla 1. Tratamientos evaluados.

\begin{tabular}{cl}
\hline Tratamiento & \multicolumn{1}{c}{ Descripción } \\
\hline TO & Testigo solo con enmienda calcárea $\left(6,6\right.$ tha $^{-1}$ Dolomita $)$. \\
T1 & Abonada comercial con 15-15-15 $\left(0,44 \mathrm{t} \mathrm{ha}^{-1}\right.$ de $\left.15-15-15\right)+$ Dolomita \\
T2 & Abonada Cachaza madura $100 \%\left(5,7 \mathrm{t} \mathrm{ha}^{-1 * *}\right)$ \\
T3 & Abonada Abimgra $100 \%\left(3,3 \mathrm{t} \mathrm{ha}^{-1 * *}\right)$ \\
T4 & Abonada $75 \%$ Cachaza madura $+25 \%$ Abimgra $\left(4,27+0,25 \mathrm{t} \mathrm{ha}^{-1}\right)$ \\
T5 & Abonada $50 \%$ Cachaza $+50 \%$ de Abimgra $\left(2,85+1,65 \mathrm{t} \mathrm{ha}^{-1}\right)$ \\
T6 & Abonada $25 \%$ Cachaza madura $+75 \%$ Abimgra $\left(1,42+2,47 \mathrm{t} \mathrm{ha}^{-1}\right)$ \\
\hline
\end{tabular}

* Fertilización química basada en la extracción por el cultivo indicador según monómeros. **Dosis calculada con base al aporte de $\mathrm{N}$ de la cachaza madura $(1,15 \%)$,

Se realizó el muestreo y caracterización de suelos un mes antes de la siembra, el cual fue clasificado como Chromic Dystruderts; el lote trabajado se encontraba en descanso los últimos 20 años antes del ensayo debido al ordenamiento de la finca; las muestras de suelos se tomaron para cada tratamiento a $20 \mathrm{~cm}$ de profundidad en toda el área del experimento con barreno holandés, las submuestras se mezclaron y se obtuvo una muestra compuesta de $1 \mathrm{~kg}$ para el análisis fisicoquímico de cada uno de los tratamientos, este procedimiento se repitió después de la cosecha del frijol, no obstante el muestreo se realizó para cada 
unidad experimental con 3 submuestras y obtener la muestra representativa; se realizó la preparación del suelo mediante arada con bueyes y dos cruzadas de arado. Luego, se realizó el trazo según el diseño experimental y se procedió a aplicar la cachaza madura de trapiche panelero, que se regó en la unidad experimental manualmente y se incorporó con azadón; la siembra se realizó un mes después de esta actividad, debido a que la cachaza debe reaccionar en el suelo y terminar de perder su capacidad fitotóxica, generada por el alto contenido de $\mathrm{CH}_{4}$ y $\mathrm{CO}_{2}$ que la enmienda presenta (Zérega 1993) al igual que la Dolomita reaccione y se logre el beneficio esperado.

Las labores agronómicas realizadas para garantizar el óptimo desarrollo de la especie se basó en control manual de arvenses; no se realizó manejo químico de plagas y enfermedades ya que el porcentaje de incidencia no justificaba su aplicación. Las variables mediadas en el ensayo fueron calcio, magnesio y potasio antes de la siembra y después de cosecha, las que se determinaron con el método de extracción por acetato de amonio 1 normal, $\mathrm{pH} 7$ y técnica de detección absorción atómica, realizados en el laboratorio de servicio a la comunidad de la Universidad Pedagógica y Tecnológica de Colombia, Tunja.

Los datos obtenidos fueron sometidos a un análisis de varianza (Anava) y se realizó la prueba de DMS, de comparación múltiple, con una confiabilidad del $95 \%$, el proceso estadístico fue realizado en el programa SmartGrafics.

\section{RESULTADOS Y DISCUSIÓN}

Se presentaron diferencias estadísticas significativas $(P<0,05)$ en las concentraciones de $\mathrm{Ca}, \mathrm{Mg}$ y $\mathrm{K}$ en la medición final, en comparación a la inicial. En general la variable calcio (Figura 1) aumentó en todos los tratamientos, presentándose los mayores

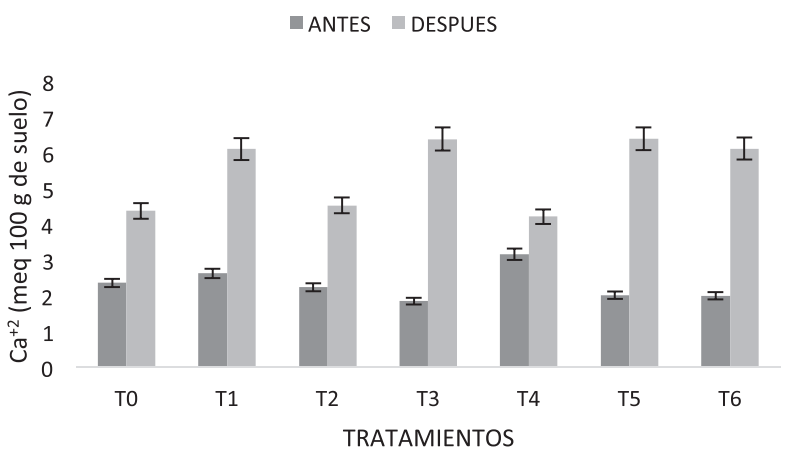

T0:Testigo solo con enmienda calcárea (6,6 t ha ${ }^{-1}$ Dolomita). T1:Abonada comercial con 15-15-15 (0,44 t ha $\mathrm{h}^{-1}$ de 15-15-15) + Dolomita; T2: Abonada Cachaza madura 100\% (5,7 t ha-1**); T3: Abonada Abimgra 100\% (3,3 $\left.\mathrm{t} \mathrm{ha}^{-1 * *}\right)$; T4:Abonada 75\% Cachaza madura $+25 \%$ Abimgra $\left(4,27 \mathrm{t} \mathrm{ha}^{-1}\right.$ más $\left.0,25 \mathrm{t} \mathrm{ha}^{-1}\right)$. T5: Abonada $50 \%$ Cachaza $+50 \%$ de Abimgra $\left(2,85 \mathrm{t} \mathrm{ha}^{-1}\right.$ más 1,65 $\left.\mathrm{t} \mathrm{ha}^{-1}\right)$; T6: Abonada 25\% Cachaza madura $+75 \%$ Abimgra $\left(1,42 \mathrm{t} \mathrm{ha}^{-1}\right.$ más $\left.2,47 \mathrm{tha}^{-1}\right)$.

Figura 1. Efecto de las bases intercambiables $\mathrm{Ca}^{+2} \mathrm{a}$ la aplicación de cachaza madura antes y después de la cosecha de frijol, Chitaraque Boyacá 2015.

valores en los tratamientos T5 $(50 \%$ Cachaza + 50\% Abimgra) seguido del T3 (100\% Abimgra) y T1 (Testigo Comercial). El aumento en las concentraciones de $\mathrm{Ca}^{+2}$ en el testigo comercial donde se realizó la aplicación de Dolomita para la corrección de la alta saturación de aluminio intercambiable, puesto que la reactividad de la enmienda al corregir la acidez intercambiable, suministra al suelo una concentración del elemento en solución del mismo, mejorando la reserva nutricional del suelo en partes de calcio y magnesio principalmente (Forero et al. 2008; Castro y Gómez 2010; Osorno 2012; Bernal et al. 2014).

Se ha reportado que el contenido de elementos mayores calcio, magnesio y potasio provenientes de la cachaza no supera la unidad (Bohórquez et al. 2014), contrario a lo encontrado en este estudio para el elemento calcio utilizando la cachaza madura, con un aporte del 6,52\%; y como se ha mencionado, la riqueza nutricional de la cachaza difiere significativamente dependiendo de las actividades internas manejadas en el trapiche, 
en el sentido estricto del método de obtención artesanal de la panela que se maneja en la Hoya del Rio Suárez; donde, se utilizan grandes cantidades de cal para el proceso de ajuste de $\mathrm{pH}$ de la sacarosa de manera empírica, por lo cual la concentración tan alta de este elemento puede provenir directamente de la cantidad de cal aplicada y su retiro posterior en la cachaza, que permite explicar el aporte significativo de este elemento en el suelo para los tratamientos T5, T1, T3, T6.

El calcio es la excepción en comparación con el magnesio y potasio, los cuales no superaron el $1 \%$ confirmando lo reportado por Bohórquez et al. (2014); sin embargo, los aportes nutricionales comerciales reportados por el producto Abimgra en lo referente a Calcio $(15,28 \%)$, Magnesio $(0,4 \%)$ y Potasio $(0,78 \%)$ tienen una tendencia similar al abono procedente del proceso panelero, por lo cual las concentraciones de calcio aumentan significativamente, convirtiéndose en un buen compensador del elemento en el suelo en estudio y se entendería que aplicados solos o en mezcla son una excelente alternativa de aporte nutricional en los suelos de la zona, los niveles óptimos de calcio se encuentran entre $\left(3-6 \mathrm{cmol}(+) \mathrm{kg}^{-1}\right)$ y los resultados obtenidos superaron este rango, lo que posiblemente generó un antagonismo con magnesio y potasio.

La adición de cachaza en el cultivo también favoreció el incremento del calcio en el suelo por el alto contenido que ésta presentó (6,52\%) y coincide con lo reportado por (Bernal y Forero 2013) quienes observaron que con la aplicación de cachaza al suelo, se aumentaron los niveles de $\mathrm{Ca}^{+2}$, y también los de $\mathrm{K}^{+}$y $\mathrm{Mg}^{+2}$. Por otro lado, se sabe que la cachaza durante su descomposición produce altas cantidades de $\mathrm{CO}_{2}$, que al transformarse en $\mathrm{H}_{2} \mathrm{CO}_{3}$, junto con otros ácidos de origen orgánico, disolverían los nutrimentos insolubles (Laura et al. 1973; Subba 1983; Swarup 1985) lo cual es muy probable debido a que en la zona de estudio predominan minerales con alto contenido de calcio como la calcita (IGAC-UPTC 2005).

Para el Magnesio, la figura 2, muestra diferencias significativas en los tratamientos, la mejor respuesta fueron el T5 $\left(2,85 \mathrm{t} \mathrm{ha}^{-1}\right.$ Cachaza madura más 1,65 t ha-1 Abimgra), seguido de los tratamientos T0 (6,6 t ha-1 Dolomita) y T2 (5,7 t ha-1 Cachaza madura), el aumento del elemento en el tratamiento testigo, se debe principalmente al uso de Dolomita utilizada para la corrección de la alta saturación de aluminio intercambiable, la cual aporta entre un $10 \%$ a un $35 \%$ de carbonato de magnesio $\left(\mathrm{MgCO}_{3}\right)$ (Castro y Gómez 2010). Esta premisa respaldaría que el aumento en las concentraciones de calcio y magnesio se debió principalmente a la reactividad de la enmienda calcárea y la solubilidad de los elementos a la fase intercambiable del suelo, luego del desplazamiento del aluminio intercambiable presente en la fase coloidal (Combatt et al. 2008); el magnesio su valor ideal esta entre $\left(1,5-2,5 \mathrm{cmol}^{(+)} \mathrm{kg}^{-1}\right)$, para el caso, solo el tratamiento T5 se ubica en un valor intermedio,

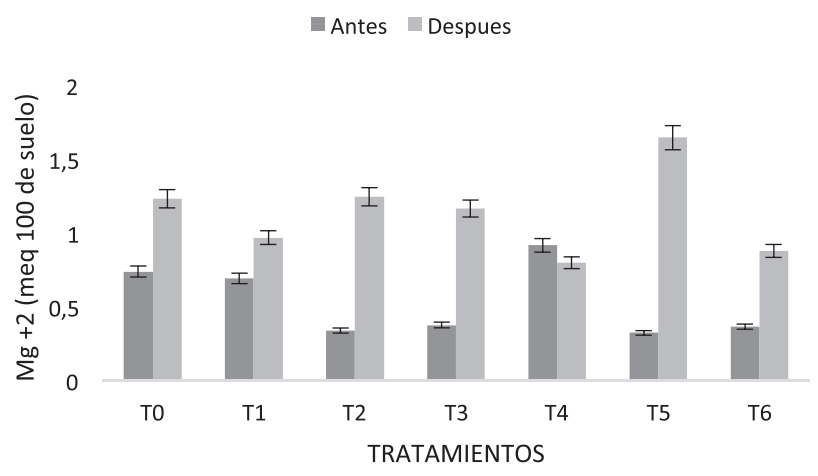

T0:Testigo solo con enmienda calcárea (6,6 t ha ${ }^{-1}$ Dolomita). T1:Abonada comercial con 15-15-15 (0,44 t ha-1 de 15-15-15) + Dolomita; T2: Abonada Cachaza madura 100\% (5,7 t ha-1**); T3: Abonada Abimgra 100\% (3,3 t ha $\left.{ }^{-1 * *}\right)$; T4:Abonada 75\% Cachaza madura $+25 \%$ Abimgra $\left(4,27 \mathrm{t} \mathrm{ha}^{-1}\right.$ más $\left.0,25 \mathrm{t} \mathrm{ha}^{-1}\right)$. T5: Abonada $50 \%$ Cachaza $+50 \%$ de Abimgra $\left(2,85 \mathrm{t} \mathrm{ha}^{-1}\right.$ más $\left.1,65 \mathrm{t} \mathrm{ha}^{-1}\right) ; \mathrm{T} 6$ : Abonada 25\% Cachaza madura + 75\% Abimgra (1,42 t ha ${ }^{-1}$ más 2,47 t ha $\left.^{-1}\right)$.

Figura 2. Efecto de las bases intercambiables $\mathrm{Mg}^{+2}$ a la aplicación de cachaza madura antes y después de la cosecha de frijol, Chitaraque Boyacá 2015. 
indicando la necesidad de complementarlo con la fertilización para evitar desbalance con el calcio y potasio.

En el caso del magnesio para los tratamientos T5 y T2, el aumento significativo del elemento se debe a la presencia del óxido de magnesio, forma en la cual su reactividad en el suelo es mayor, evidenciándose en el aumento significativo de la base en la fase coloidal (Montaño y Forero 2013).

Las concentraciones de potasio (Figura 3) mostraron un aumento en todos los tratamientos especialmente en el tratamiento T1 (Testigo comercial), seguido de los tratamientos a los cuales se les aplicó Cachaza y Abimgra solos y en mezcla, debido a que el contenido del elemento en las fuentes orgánicas utilizadas son muy bajos (Figura 1C); las altas concentraciones de $\mathrm{K}^{+}$pudo deberse a la disponibilidad del elemento de una forma más eficiente presente en el fertilizante químico. Zérega (1993) afirma, que con los aportes de potasio por parte de la cachaza, se requiere la aplicación alterna del

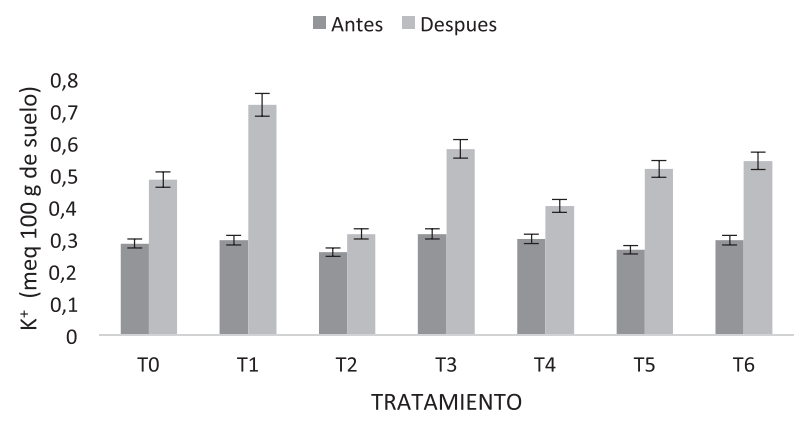

T0:Testigo solo con enmienda calcárea $\left(6,6 \mathrm{t} \mathrm{ha}^{-1}\right.$ Dolomita). T1:Abonada comercial con 15-15-15 (0,44 t ha-1 de 15-15-15) + Dolomita; T2: Abonada Cachaza madura 100\% (5,7 t ha-1**); T3: Abonada Abimgra 100\% (3,3 $\left.\mathrm{t} \mathrm{ha}^{-1 * *}\right) ; \mathrm{T} 4$ :Abonada 75\% Cachaza madura + 25\% Abimgra (4,27 tha ${ }^{-1}$ más 0,25 t ha $\left.^{-1}\right)$. T5: Abonada $50 \%$ Cachaza $+50 \%$ de Abimgra $\left(2,85 \mathrm{t} \mathrm{ha}^{-1}\right.$ más $\left.1,65 \mathrm{t} \mathrm{ha}^{-1}\right) ; \mathrm{T} 6$ : Abonada 25\% Cachaza madura + 75\% Abimgra (1,42 t ha ${ }^{-1}$ más 2,47 t ha $\left.^{-1}\right)$.

Figura 3. Efecto de las bases intercambiables $\mathrm{k}^{+}$a la aplicación de cachaza madura antes y después de la cosecha, Chitaraque Boyacá 2015. elemento para suplir las necesidades de las plantas, especialmente cuando se aplican en suelos con texturas arenosas.

A pesar que la liberación de nutrientes de la materia orgánica es más lenta que los fertilizantes minerales por depender de la mineralización de ésta (Campo 1996), el K+ por no encontrarse ligado a ningún compuesto orgánico, se libera inmediatamente después de la aplicación del material orgánico, en este caso de la cachaza (Damatto et al. 2006).

Zérega (1993) afirma que la cachaza aumenta el $\mathrm{pH}$ del suelo; debido a alto contenido de carbonato de calcio (Prasad 1976; Painulí 1986); Tambien, De Camargo et al. (1984) sostienen que la cachaza tiene un poder de neutralización del $25 \%$ respecto al $\mathrm{CaCO}_{3}$. Además se ha encontrado que corrige el aluminio tóxico. El aumento del $\mathrm{pH}$ del suelo influye en la liberación del $\mathrm{K}^{+}$no intercambiable, y en consecuencia también lo hace en el equilibrio entre esta fracción y él. Se sabe que una elevación del $\mathrm{pH}$ y la presencia de calcio en la solución del suelo favorecen la liberación del $\mathrm{K}^{+}$atrapado entre las unidades cristalinas de las arcillas, ya que el $\mathrm{Ca}^{+2}$ al tener un volumen mayor que el $\mathrm{K}^{+}$cuando se introduce entre los espacios interlaminares separa estos, permitiendo la salida del K+ (Navarro y Navarro 2003).

Tal como lo explica Arreche (2008) la necesidad de realizar aplicaciones de cualquier enmienda orgánica, radica en determinar el potencial nutricional de la misma en este sentido, las concentraciones de nutrientes en la cachaza varían según el proceso de clarificado de la panela, entonces el mejoramiento nutricional de la misma puede ser manejado desde el proceso productivo en trapiche, requiriendo una necesidad de perfeccionamiento del sistema para que el insumo sea de alto potencial productivo. 
Las condiciones presentadas en este estudio, concuerdan con lo expresado por Soto (2003) y Sosa (2005), los cuales afirman que las aplicaciones de cachaza madura aumenta el contenido de calcio, magnesio y potasio en suelo, con la activación de la actividad de los microorganismos del suelo, los cuales están relacionada íntimamente con el proceso de mineralización de la materia orgánica, respuesta que respalda la tendencia a la degradación directa del compuesto Abimgra cuando es mezclado con cachaza como lo relaciona Zambrano (2005), los estiércoles aplicados en mezcla con productos con altos contenidos de azúcares (como la cachaza) aceleran el proceso de liberación de elementos nutricionales.

\section{CONCLUSIONES}

La aplicación de cachaza madura sola o en mezcla aumenta los contenido de calcio y magnesio, siendo este material una opción favorable para el sistema de producción panelero de la zona de la Hoya del Rio Suárez (Chitaraque, Boyacá), como sustituto de otras enmiendas.

Las concentraciones de potasio en el suelo aumentaron con la aplicación de cachaza, sin embargo, las concentraciones son superiores con las aplicaciones de fertilizantes químicos, lo que indicaría que la fertilización del elemento debe hacerse conjugando la cachaza con un abono de síntesis química.

\section{REFERENCIAS}

Arreche, I. 2008. Efecto de la fertilización orgánica y química en suelos degradados cultivados con Maíz (Zea maiz L.) en el estado Yacury, Venezuela. Tesis Doctoral. Universidad de Valladolid. España. 21.
Arreola-Enríquez, J., Palma-López, D., SalgadoGarcía, S., Camacho-Chiw, W., ObradorOldan, J., Juárez- López, J. y PastranaAponte, J. 2004. Evaluación del abono orgánico- mineral de cachaza en la producción y calidad de la caña de azúcar. Terra latinoamerica. 22(3):351-357.

Bernal, A. y Forero F. 2013. Evaluación de especies vegetales para el manejo de la acidez en suelos Sulfatados ácidos de Paipa- Boyacá. Corpoica Ciencia y Tecnología. 15(2):229-236.

Bernal, A., Montaño, J., Sánchez, R., Albarrán, V. y Forero, F. 2014. Efecto de las diferentes dosis de materiales encalantes y orgánicos sobre las bases intercambiables de un suelo sulfatado ácido en invernadero. Temas agrarios. 19(1): 19-31.

Bohórquez, A., Puentes, Y. y Menjivar, J. 2014. Evaluación de la calidad del compost producido a partir de subproductos agroindustriales de caña de azúcar. Revista Corpoica Ciencia y Tecnología Agropecuaria.15(1): 73-81.

Campo, F. 1996. Frutas de clima temperado II: Figo, maçã, marmelo, pêra e pêssegoem pomar compacto. In: RAIJ, B. van. et al. (Ed.). Recomendações de adubação e calagem para o Estado de São Paulo. 2.ed. Campinas:Fundação Instituto Agronômico. 139-140.

Castro, H. y Gómez, I. 2010. Fertilidad y fertilizantes. Editores Burbano $\mathrm{H}$, Silva F. Ciencia del suelo, Principios básicos. Sociedad Colombiana de Colombiana de la Ciencia del Suelo. Bogotá D.C.89.

Cifuentes, R., De Leon, R.y Porres, C. 2011. Producción de abono orgánico a partir de cachaza y tallos de caña de azúcar recuperados de las carreteras. Revista 23 de la Universidad del Valle de Guatemala. 8-17. 
Combatt, E., Jarma, A. y Daza, L. 2008. Crecimiento de Brachiaria de cumbensstapf y Cynodonn lem fuensis vanderyst en suelos sulfatados ácidos de Córdoba. Revista MVZ Córdoba. 13(2): 1380-1392.

Damatto, J., Roberto, V. Sarita, L. y Dirceu, M. 2006. Alteraçõesempropriedades de solo adubadocom doses de compostoorgânicosob cultivo de bananeira Rev. Bras. Frutic. 28(3).

De Camargo, A., Berton, S., Geraldi, R. y Aires, J. 1984. Alterações de aracterísticas químicas de um latossol oroxodistrófico incubado comresíduos da industria álcool-açucareira. BRAGANTIA. 43(1), 125-139.

Forero, F. Torres, J. y Balaguera, H. 2008. Efecto de la aplicación de cachaza fresca y de dos sistemas de producción, Maíz y Maíz asocio frijol sobre las propiedades físicas de un Inceptisol. Revista Colombiana de Ciencias Hortícolas. 2(2): 205-216.

Gómez, J. 2006. Bioabono de la cachaza. Proyecto SICA. Banco Mundial. Ministerio de Agricultura del Ecuador. 2.

Gómez. P. y Jaramillo. L, 2006. Estudio de factibilidad para el uso de cachaza generada a partir del proceso de la caña de azúcar como abono. Universidad de la Sabana. Bogotá D.C. 42 p.

Hugot, E. and Jenkins, G. 1972. Handbook of Cane Sugar Engineering. New York: Elsevier. 861-88.

\section{Instituto Geográfico Agustín Codazzi,} Universidad Pedagógica y Tecnológica De Colombia (UPTC). 2005. Estudio general de suelos y zonificación de tierras del departamento de Boyacá. Bogotá D.C. Tomo I y Tomo II. 547.
Laura, R. AndIdnani,A. 1973. A study on the ameliorative effects of addition of farm compost, charcoal dust and sawdust in saline and alkali soil. Agrochimica. 17(5), 480-88.

Mohee, R. and Panray, B. 1999. Life cycle analysis of compost incorporated sugarcane bioenergy systems in Mauritius Biomass and Bioenergy 17. 73-83.

Montaño, J. y Forero F. 2013. Subproductos orgánicos del proceso panelero en la recuperación de Suelos sulfatados ácidos a nivel de Invernadero. Tesis de Maestría Ingeniería Ambiental. Universidad Pedagógica y Tecnológica de Colombia. 145.

Navarro, G. y Navarro, S. 2003. Química agrícola. Ed Mundi prensa. España. 564.

Osorno. H. 2012. Mitos y realidades de las Cales y las enmiendas de Colombia. Tesis Doctoral. Universidad Nacional de Colombia. Sede Medellín. 187.

Painuli, K. andAbrol,P. 1986. Role of some organics in improving sodic soils. Indian J. Agríe. Sel. 56(4): 267-76.

Prasad, M.1976. Response of sugarcane to filter press mud and $\mathrm{N}, \mathrm{P}$, and $\mathrm{K}$ fertilizar. II. Effects on plant composition and soil chemical properties. Agron. J. 68(4): 543574.

Sosa, O. 2005. Los estiércoles y el uso como enmiendas orgánicas. Agromensajes. 16(2):30-34.

Soto, M. 2003. Abonos orgánicos: definiciones y procesos. En: Melendez G. (Eds.) Abonos Orgánicos: principios, aplicaciones e impactos en la agricultura. San José de Costa Rica. 20-49. 
Subba, M. 1983. La cachaza. Monografía del grupo de Países Latinoamericanos y del Caribe exportadores de azúcar GEPLACEA. 22.

Swarup,A. 1985. Yiel and nutrition of rice as influenced by Presubmergence and amendments in a highiysodic soil. J. Indian Soc. Sel. 33(2): 352-57.

Treto, E. 2005. Avances en el manejo de los suelos y la nutrición orgánica. Instituto Nacional de Ciencias Agrícolas. Universidad Nacional de la Habana Cuba. 20: 2-19.
Zambrano. A. 2005. Caracterización química de enmiendas orgánicas. Tesis de Maestría. Universidad Central de Venezuela. 92.

Zérega. L. 1993. Manejo y uso agronómico de la cachaza en suelos cañameleros- Caña de azúcar. 11(2). 18.

Zurro, P. 2005. Producción de abonos y fertilizantes a partir de subproductos de la industria azucarera orgánica, En: http://www.elparanaense.com.ar/pd f/ memorasabonosIngSanJavierMNES.pdef. 4p. Consulta 16 Mayo 2016. 